\title{
Development of a FLIPR Assay for the Simultaneous Identification of MrgD Agonists and Antagonists from a Single Screen
}

\author{
Seena K. Ajit, ${ }^{1,2}$ Mark H. Pausch, ${ }^{2}$ Jeffrey D. Kennedy, ${ }^{2}$ and Edward J. Kaftan ${ }^{2}$ \\ ${ }^{1}$ Department of Pharmacology and Physiology, Drexel University College of Medicine, 245 North 15th Street, MS number 488, \\ Philadelphia, PA 19102, USA \\ ${ }^{2}$ Neuroscience Discovery, Pfizer Global Research and Development, CN 8000, Princeton, NJ 08543, USA
}

Correspondence should be addressed to Seena K. Ajit, seena.ajit@drexelmed.edu

Received 1 April 2010; Revised 8 July 2010; Accepted 6 August 2010

Academic Editor: John V. Moran

Copyright ( $) 2010$ Seena K. Ajit et al. This is an open access article distributed under the Creative Commons Attribution License, which permits unrestricted use, distribution, and reproduction in any medium, provided the original work is properly cited.

\begin{abstract}
MrgD, a member of the Mas-related gene family, is expressed exclusively in small diameter IB4 ${ }^{+}$neurons in the dorsal root ganglion. This unique expression pattern, the presence of a single copy of MrgD in rodents and humans, and the identification of a putative ligand, beta-alanine, make it an experimentally attractive therapeutic target for pain with limited likelihood of side effects. We have devised a high throughput calcium mobilization assay that enables identification of both agonists and antagonists from a single screen for MrgD. Screening of the Library of Pharmacologically Active Compounds (LOPAC) validated this assay approach, and we identified both agonists and antagonists active at micromolar concentrations in MrgD expressing but not in parental CHO-DUKX cell line. Further characterization was performed using a subset of these screening hits. Our results demonstrated that the dual agonist/antagonist assay format is feasible and likely can be extended to most GPCRs with known agonist.
\end{abstract}

\section{Introduction}

The seven transmembrane G-protein coupled receptors (GPCRs) comprise one of the largest gene families in the human genome and represent an estimated $25 \%$ of all drug targets [1]. They are activated by a variety of molecules including, but not limited to, neurotransmitters, peptides, lipids, odorants, and light, and thus participate in a wide range of physiological responses. Reverse pharmacology strategies are usually employed in the identification of ligands for newly identified GPCRs. These ligands are in turn used for the pharmacological characterization and identification of the physiological role of these receptors [2].

Recent studies have identified a GPCR subfamily predominantly expressed in small diameter IB4 ${ }^{+}$neurons in the dorsal root ganglion (DRG) and as such, might have a role in nociception. Members of this family have been referred to as Mas-related genes (Mrgs) [3] or sensory neuron specific receptors (SNSRs) [4]. In mice, the Mrg family is comprised of three large subfamilies (MrgA, MrgB, and $\operatorname{MrgC}$ ) and six single copy genes ( $\mathrm{MrgD}, \mathrm{MrgE}, \mathrm{MrgF} / \mathrm{RTA}, \mathrm{MrgG}$, $\mathrm{MrgH} / \mathrm{GPR} 90$, and MAS1), that together comprise $\sim 50$ distinct sequences [3]. The functional significance of this cellular heterogeneity among murine nociceptive sensory neurons is currently not known. In contrast, there are only four functional MrgX/SNSR genes in humans; however, none of the human $\mathrm{MrgX}$ and mouse $\mathrm{MrgA}, \mathrm{B}$, or $\mathrm{C}$ genes are strictly orthologous, making investigation of their function or testing of compounds in relevant rodent models difficult. Importantly, the single copy genes $\mathrm{MrgD}, \mathrm{MrgE}, \mathrm{MrgF}$, and MrgG have clearly defined human, mouse, and rat orthologs and thus may represent experimentally tractable targets for the development of pain therapies $[3,5]$.

Though many Mrg family members are classified as orphan receptors, ligands for a number of these receptors have been identified, and are being used as tools to characterize their role in nociception. These receptor/ligand pairs include human $\mathrm{MrgX2} /$ cortistatin [6], human MrgX1 (SNSR4), SNSR3, and rat MrgC/BAM22 (bovine adrenal medulla peptide) $[4,7]$, and MrgA1, MrgA4, and 
MrgC11/RF-amide neuropeptides [3, 8]. Beta-alanine was identified as a ligand for $\mathrm{MrgD}$, specifically evoking an intracellular $\mathrm{Ca}^{2+}$ response in CHO cells expressing human, rat, or mouse MrgD [9]. Grazzini et al. studied nociception resulting from the activation of rat $\mathrm{MrgC}$ by its ligand BAM22 [7]. Selective MrgC agonists produced spontaneous pain behavior suggesting that an antagonist of this receptor may be of therapeutic value in treating pain. A cell-based beta-lactamase (BLA) reporter gene assay to identify small molecule antagonists of the human MRGX1 receptor also has been reported [10]. Though beta-alanine has been identified as a putative agonist for MrgD [9], studies have not been reported describing its effects on pain.

By virtue of its cross-species conservation as a singlecopy gene as well as its restricted expression to small diameter nociceptive neurons, MrgD represents an attractive target for the development of pain therapeutic agents, an endeavor that would be facilitated by the identification of potent agonists and antagonists. A FLIPR based screen for MrgA and $\mathrm{MrgD}$ agonists [11] has been published recently, but no method for identifying $\mathrm{MrgD}$ antagonist has been yet reported. The objective of the current study was to develop a $\mathrm{MrgD}$ assay amenable to high throughput screening (HTS) that is capable of simultaneously identifying agonists and antagonists. Screening compound libraries in this assay format could be useful in the identification of tool compounds to probe the physiological role(s) of MrgD.

\section{Materials and Methods}

2.1. Chemicals and Reagents. Beta-alanine, GABA, glycine, and the LOPAC ${ }^{640}$ library were obtained from Sigma (St. Louis, MO). A MrgX1 (SNSR4) cell line was purchased from Multispan (Hayward, CA). All cell culture reagents were from Invitrogen (Carlsbad, CA).

2.2. MrgD Stable Cell Line Generation. Human MrgD (Accession number AY427820) was amplified from human genomic DNA (Clontech, Palo Alto, CA) by PCR using the forward primer Xbal $5^{\prime}$-CTAG TCT AGA GCC GCC ACC ATG TAC CCA TAC GAT GTT CCA GAT TAC GCT AAC CAG ACT TTG AAT AGC AGT-3' and the reverse primer EcoR1 5'-CCG GAA TTC TCA AGC CCC CAT CTC ATT GGT-3'. A Kozak sequence and HA epitope tag were incorporated in the forward primer. Rat MrgD (Accession number AF518246) was amplified and cloned similarly using the forward primer Xbal $5^{\prime}$-CTAG TCT AGA GCC GCC ACC ATG GAA CAA AAA CTT ATT TCT GAA GAA GAT CTG AAC TAC ACT CCT TAT AGC AGC C-3' and the reverse primer EcoR1 5' -CCG GAA TTC TCA GAC CCC ATC ATT AGT ACA TGT-3' using rat genomic DNA (Clontech, Palo Alto, CA) as template. A Kozak sequence and c-Myc epitope tag were incorporated in the forward primer. The resulting PCR products were cloned into the Xba I and EcoR I sites of pHTOP vector, a proprietary derivative of the pED expression vector [12]. For transfection, CHO-DUKX-A2 cells (DHFR(-) CHO cell line stably expressing the tetracycline transactivator) were grown in $\mathrm{M}$ alpha media containing 1\% MEM nonessential amino acids solution (NEAA), 10\% fetal bovine serum (FBS), and $500 \mathrm{ug} / \mathrm{mL}$ G418. MrgDpHTOP was transfected using Lipofectamine Plus reagents as per the manufacturer's recommendations. Antibiotic selection was performed using Dulbecco's modified Eagle medium supplemented with 10\% FBS, 100 units $/ \mathrm{mL}$ penicillin, $100 \mu \mathrm{g} / \mathrm{mL}$ streptomycin, $1 \%$ NEAA, and $20 \mathrm{nM}$ methotrexate (Calbiochem, San Diego, $\mathrm{CA})$. Cell lines were maintained at $37^{\circ} \mathrm{C}$ in a humidified atmosphere containing $5 \% \mathrm{CO}_{2}$. Surviving colonies were expanded further and used for functional analysis. Clones of human MrgD expressing cell lines were evaluated initially for MrgD transcript level by Taqman analysis and normalized against the house-keeping gene GAPDH (Glyceraldehyde-3phosphate dehydrogenase).

2.3. $\left[\mathrm{Ca}^{2+}\right]$ FLIPR Assays. CHO-DUKX-A2 cells stably expressing human or rat MrgD were plated in 384-well tissue-culture-treated polystyrene black clear flat-bottom assay plates (BD Biosciences, San Jose, CA). Thirty $\mu \mathrm{L}$ of $8 \times 10^{5} \mathrm{cells} / \mathrm{mL}(24,000$ cells/well $)$ were plated and maintained for $\sim 24 \mathrm{hrs}$ at $37^{\circ} \mathrm{C}$ in a humidified atmosphere containing $5 \% \mathrm{CO}_{2}$. After removal of the growth medium, cells were incubated with $30 \mu \mathrm{L}$ of $\mathrm{Ca} 3$ dye (Molecular Devices Corporation, Sunnyvale, CA) containing $2.5 \mathrm{mM}$ freshly prepared probenecid made according to the manufacturer's protocol. Agonists were prepared in 1x Hanks balanced salt solution (HBSS) buffer with $20 \mathrm{mM}$ HEPES buffer. Changes in intracellular $\mathrm{Ca}^{2+}$ were monitored using the Fluorometric Imaging Plate Reader (FLIPR; Molecular Devices, Sunnyvale, CA). For the double addition protocols, compounds were added by the FLIPR, and readings were taken for $3 \mathrm{~min}$, followed by the addition of the agonist. The change in intracellular calcium $\left[\mathrm{Ca}^{2+}\right]$ was monitored as an increase in relative fluorescence units (RFUS), and the difference between maximum and minimum RFU was used to calculate dose-response curves. Data analysis was performed using PRISM 4.0 (GraphPAD Software Inc.). For the LOPAC screen, cells were plated in 96-well tissue-culturetreated polystyrene black clear flat-bottom assay plates (BD Biosciences, San Jose, CA). One hundred $\mu L$ of cells $(40,000$ cells/well) were plated and maintained under the same conditions as above. For the assay, growth medium was replaced with $90 \mu \mathrm{L}$ of Ca3 dye containing $2.5 \mathrm{mM}$ freshly prepared probenecid. The FLIPR added $10 \mu \mathrm{L}$ of beta-alanine at $100 \mu \mathrm{M}$ final concentration. For antagonist dose-response assays, the cells were incubated with the compounds for $\sim 30$ minutes after which beta-alanine at a final concentration of $10 \mu \mathrm{M}$ was added by the FLIPR.

\section{Results}

3.1. Characterization of Human and Rat MrgD Cell Lines. Clones of human MrgD expressing cell lines were expanded and evaluated initially for MrgD transcript level by Taqman analysis. MrgD transcripts were detected in 25 clones displaying wide-ranging expression levels (data not shown). Eleven clones spanning this expression range were selected 


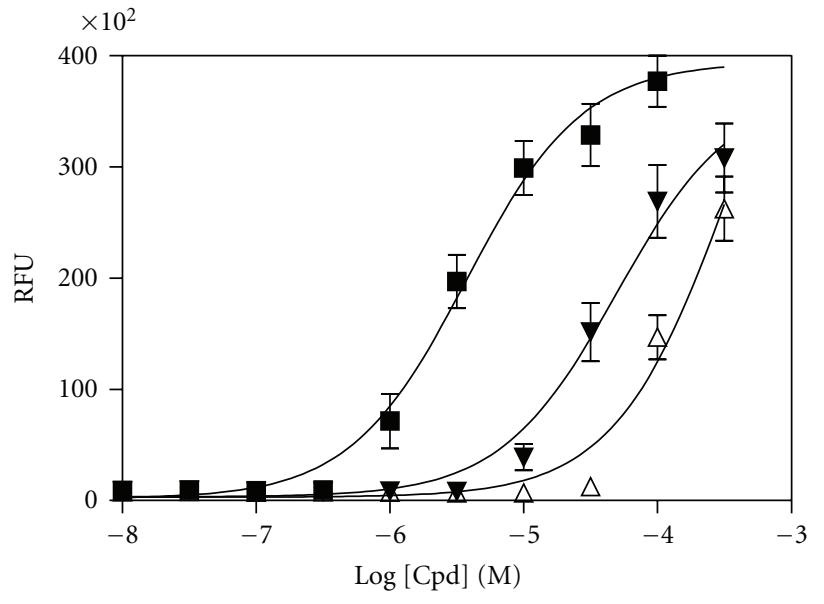

- Beta alanine
GABA

$\triangle$ Glycine

FIgure 1: Concentration response for beta-alanine, GABA, and glycine on rat $\mathrm{MrgD}$ receptor in CHO-DUKX cells. Representative data from calcium mobilization FLIPR assay performed in 384 wells are shown. Each data point represents mean of 32 replicates, and error bar represents SD. Human MrgD expressing CHO-DUKX cells also had similar dose response (data not shown).

for FLIPR studies. The parental cell line was also evaluated, and MrgD transcript was absent in CHO-DUKX cells. Surprisingly, two clones, one with medium and the other with low transcript levels, responded best to beta-alanine, while three clones with high MrgD transcript levels showed no response. Thus, our studies indicate that MrgD RNA expression inversely correlated with larger beta-alanineinduced calcium transient functional responses as assessed by FLIPR responses (data not shown). Hence, when cell lines expressing rat MrgD were generated, they were screened for responses to beta-alanine only by FLIPR. The beta-alanine $\mathrm{EC}_{50}$ values obtained for both human and rat MrgD cell lines were in the $1-4 \mu \mathrm{M}$ range. Further characterizations of $\mathrm{MrgD}$ receptor-expressing cell lines were performed by evaluating responses to ligands structurally similar to betaalanine, such as GABA and glycine. Figure 1 shows agonist dose-response curves indicating that beta-alanine is the most potent ligand followed by GABA and glycine, respectively. The $\mathrm{EC}_{50}$ values of beta-alanine obtained for human and rat $\mathrm{MrgD}$ are displayed in Table 1. In light of their structural similarity to GABA, both gabapentin and pregabalin were evaluated by FLIPR, but these drugs failed to induce a response in either the human or rat MrgD expressing cells.

\subsection{Evaluation of $G i$ and $G q$ Coupling of MrgD Receptors} Using FLIPR. MrgD is known to couple through both $\mathrm{Gi}$ and Gq [9]. To further explore the utility of the cell line for a potential secondary assay measuring cAMP, we investigated whether the FLIPR assay was capable of distinguishing the extent of Gi and Gq coupling of MrgD. MrgD CHO-DUKX cells were treated with either the Gi inhibitor pertussis toxin
TABLE 1: $\mathrm{EC}_{50}$ values obtained for MrgD agonists. Data represents mean \pm standard deviation from 2-6 independent experiments.

\begin{tabular}{lcc}
\hline \multirow{2}{*}{ Ligand } & \multicolumn{2}{c}{$\mathrm{EC}_{50}$ in $\mu \mathrm{M}$} \\
\hline Beta-alanine & $3.76 \pm 1.27$ & Rat MrgD \\
GABA & $53 \pm 26$ & $3.77 \pm 0.87$ \\
Glycine & $>100$ & $49.8 \pm 1.5$ \\
Gabapentin & No effect & $>100$ \\
Pregabalin & No effect & No effect \\
\hline
\end{tabular}

(PTX) overnight or the phospholipase C (PLC) inhibitor U-73122 for 30 or 60 minutes and then evaluated for their responses to beta-alanine. U-73122 completely blocked the FLIPR response (Figure 2(a)) but treatment with PTX resulted in approximate $50 \%$ reduction in calcium mobilization (Figure 2(b)), suggesting that the residual beta-alanineinduced response in CHO-DUKX cells is transduced through the coupling of MrgD to a PTX-insensitive G protein, most likely Gq.

3.3. Assay Development and LOPAC Library Screen. We developed an assay that could allow both agonists and antagonists to be distinguished in a single screen. Employing such an assay format should enable the identification of tool compounds that may help in elucidating the role of MrgD in pain. The diagram in Figure 3 describes the dual-addition assay format where addition of the test compound (addition 1 ) is followed by the addition of beta-alanine (addition 2). A FLIPR response immediately upon the addition of a test compound would indicate its activity as an agonist. On the other hand, if the compound behaves as an antagonist, not only will there be a lack of response to this test compound, it will also attenuate the effects of the subsequent addition of beta-alanine. In the event of no receptor engagement by the test compound, a normal agonist response will be observed to beta-alanine. We validated this assay format, which was robust and consistent with a $Z^{\prime}$ factor greater than 0.7 .

To confirm that the dual-addition FLIPR assay format can be extended to a HTS platform, a screen of the LOPAC library was performed (Figures 4(a) and 4(b) show representative FLIPR traces). Initial evaluation of the results from the dual assay format indicated that the assay was sensitive enough to identify both agonists and antagonists. The response to test compound GABA in the LOPAC screening is shown in Figure 4(a) (row G, column 6). GABA evoked a response, but as it is less potent than beta-alanine, some calcium mobilization also was induced in response to beta-alanine, albeit weaker when compared to other wells. On the other hand, when the first test compound was an antagonist occupying the MrgD receptor, no initial effect was detected, and there was an attenuation or complete absence of calcium mobilization following addition of betaalanine (C3, G4, G7, and E11-Chlorpromazine hydrochloride, $\mathrm{R}(-)$-Propyl-norapomorphine hydrochloride, indatraline hydrochloride and, thioridazine hydrochloride, resp., in Figure $4(\mathrm{~b}))$. Screening the library in parallel on parental 


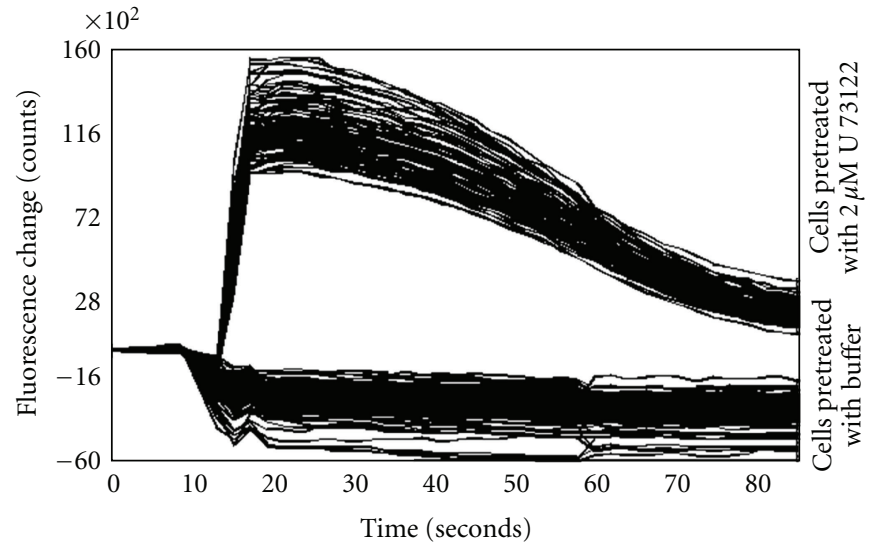

(a)

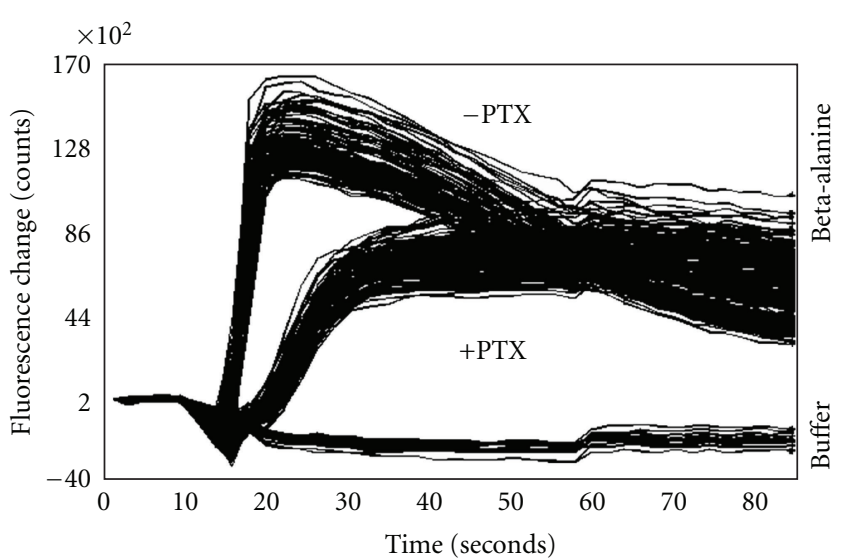

(b)

FIGURE 2: Evaluation of Gq (Figure 2(a)) and Gi (Figure 2(b)) coupling of MrgD receptors assessed using FLIPR response. MrgD cells were pretreated with the PLC inhibitor (U-73122 at $2 \mu \mathrm{M}$ for 30 or 60 minutes) or pertussis toxin (100 ng overnight) for blocking Gi coupling to MrgD. FLIPR responses were evaluated by the addition of $100 \mu \mathrm{M}$ beta-alanine.

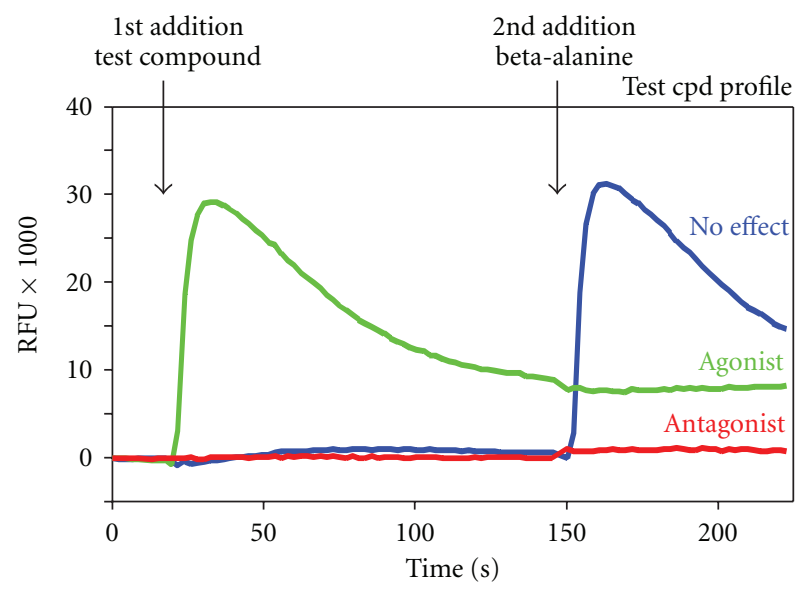

Figure 3: Assay format for the identification of an agonist and antagonist from a single screen. Test compound is added first followed by a second addition of beta-alanine. An agonist will induce a FLIPR response immediately upon the addition of a test compound. An antagonist will cause a pharmacological blockade of the MrgD receptor resulting in attenuation or complete lack of response to beta-alanine. In the event of no receptor engagement, a normal agonist response will be observed from beta-alanine addition.

CHO-DUKX cells helped in identifying compounds causing nonspecific calcium mobilization (example seen in Figure 4(b), H5). Eliminating compounds that induced calcium mobilization in the parental line ensured that the agonists identified interact specifically with $\mathrm{MrgD}$. To evaluate whether this assay format could be extended to other GPCR family members, we tested MrgX1 (SNSR4) cell lines and performed a similar dual-addition format LOPAC screen using its ligand BAM22 to identify antagonists. Our results demonstrated that this assay format could be
TABLE 2: Compounds exhibiting agonism at the MrgD receptor and not the parental CHO-DUKX cells.

\begin{tabular}{lcc}
\hline \multirow{2}{*}{ Agonist } & \multicolumn{2}{c}{$\mathrm{EC}_{50}$ in $\mu \mathrm{M}$} \\
& $\begin{array}{c}\text { Human } \\
\text { MrgD }\end{array}$ & $\begin{array}{c}\text { Rat } \\
\text { MrgD }\end{array}$ \\
\hline 5, 7 dihydroxytryptamine creatinine sulfate & 5.2 & 9.7 \\
Xanthine amine congener & $>100$ & 48.3 \\
( \pm ) HA 966 & 11.0 & 13.2 \\
\hline
\end{tabular}

applied to another GPCRs with a known agonist (data not shown).

In addition to GABA, three other agonists that stimulated MrgD expressed in the CHO-DUKX cell line but not the parental cell line were obtained from the LOPAC library screen (Table 2). One of these, $( \pm)$ HA 966 is a racemic mixture, and so the enantiomers were tested separately. As displayed in Figure 5(a) the (-) HA 966 enantiomer was the most potent $\mathrm{MrgD}$ agonist $\left(\mathrm{EC}_{50}\right.$ $5.5 \mu \mathrm{M})$ when compared to the racemic mixture $\left(\mathrm{EC}_{50}\right.$ $11 \mu \mathrm{M})$ and (+) HA 966 enantiomer (EC $50106 \mu \mathrm{M})$. In addition to agonists, eight $\mathrm{MrgD}$ antagonist compounds were also identified from the LOPAC screen. Though compounds representing eight different structural classes were identified from the LOPAC library screen, only three dopaminergic compounds chlorpromazine hydrochloride ( $\mathrm{IC}_{50}$ 2.73E-06), thioridazine hydrochloride ( $\mathrm{IC}_{50} 1.06 \mathrm{E}-$ 06 ), and $\mathrm{R}(-)$-Propylnorapomorphine hydrochloride ( $\mathrm{IC}_{50}$ 1.46E-06) could be confirmed by dose response analysis. Two representative compounds thioridazine and chlorpromazine are shown in Figure 5(b). The percentage of hits confirmed after concentration response followup assay evaluation were $0.6 \%$ and $0.4 \%$ for agonists and antagonists, respectively (Table 3). 

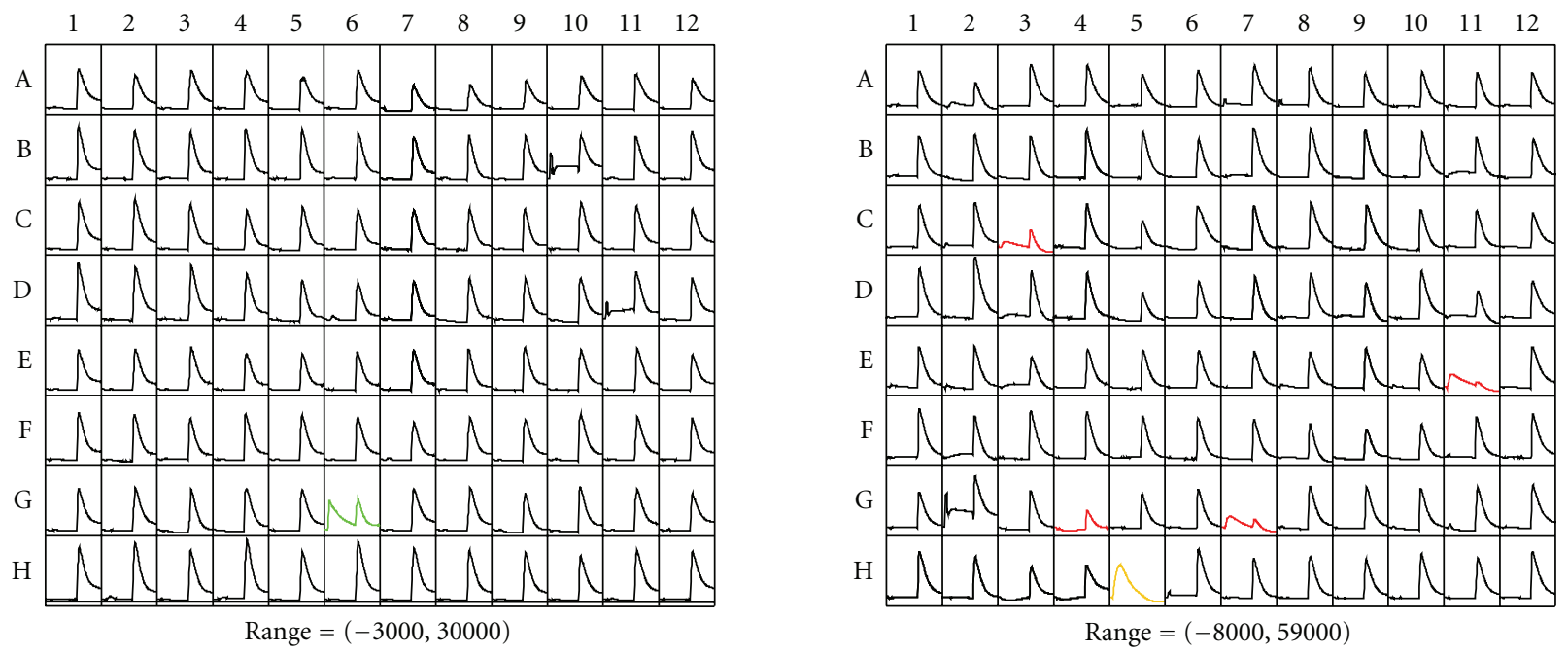

FIGURE 4: Representative FLIPR traces obtained from LOPAC screen. Compounds from the LOPAC library were applied followed by the addition of beta-alanine. Similar responses were observed for human and rat MrgD cell lines. Parental CHO-DUKX cell lines were also tested to identify nonspecific effects of test compounds. Green, red, and yellow traces represent agonist, antagonist, and nonspecific traces, respectively.

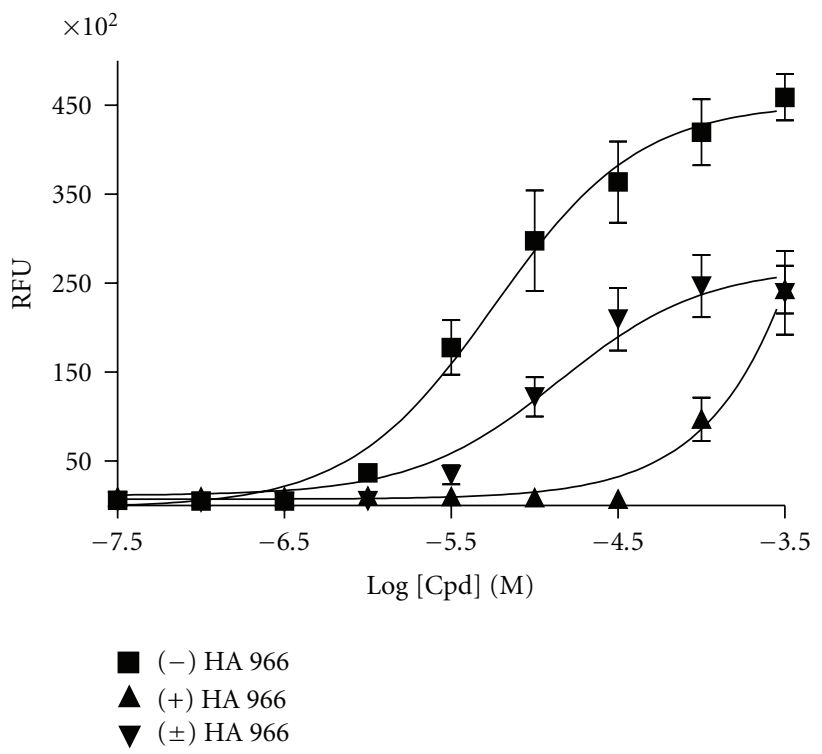

(a)

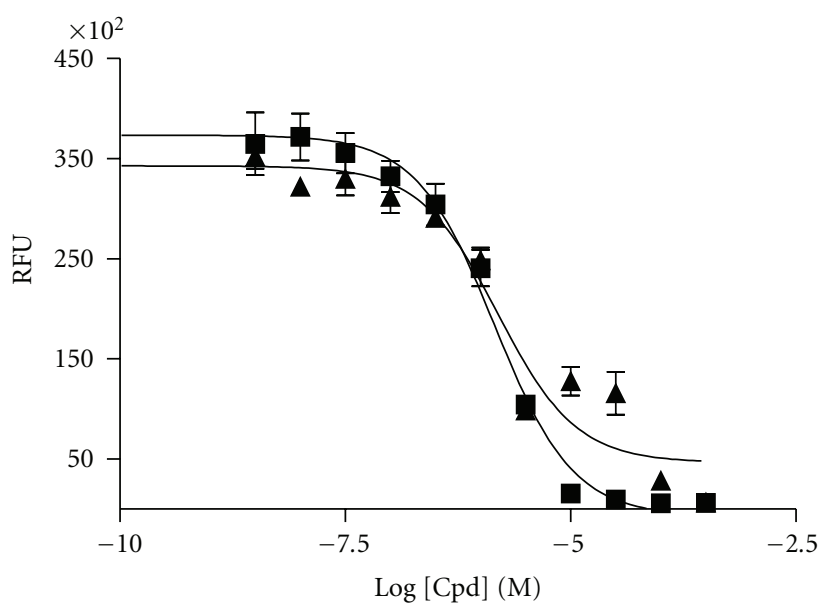

Chlorpromazine

$\boldsymbol{\Delta}$ Thioridazine

(b)

FIGURE 5: Illustration of the dose-response curve of selected agonists and antagonists. An agonist identified, ( \pm ) HA 966, is a racemic mixture, and further characterization of the positive and negative enantiomers showed that the latter was the most potent MrgD agonist among all three (Figure 4(a)). The $\mathrm{EC}_{50}$ values were 11, 106, and $5.5 \mu \mathrm{M}$ for the racemic mixture, positive, and negative isoforms of the compound, respectively. Figure 4(b) shows two representative MrgD antagonists thioridazine and chlorpromazine, with $\mathrm{IC}_{50}$ values of 1.5 and $2.7 \mu \mathrm{M}$, respectively.

\section{Discussion}

An inherent difficulty in the pursuit of novel therapeutic targets is the lack of molecular and/or pharmacological tools. Several approaches are usually employed, often in parallel, to obtain insight into the functional role of novel receptors in disease states. The scientific rationale for pursuing drug discovery efforts is often supported by the degree to which a particular target is amenable to available drug discovery technologies, its tissue localization, changes in gene expression, availability of gain or loss of function transgenic animals, and the availability of tool compounds. Receptor-ligand interactions vary, and ligands can function as agonists, antagonists, partial antagonists, 
TABle 3: Structural class of compounds obtained from LOPAC library screen that were confirmed in dose-response FLIPR assay.

\begin{tabular}{lcc}
\hline & Structural class & $\begin{array}{c}\text { Percentage of } \\
\text { confirmed hits }\end{array}$ \\
\hline Agonists & $\begin{array}{c}\text { Serotonergics, glutamatergics, } \\
\text { adenosine purinergic, and } \\
\text { Gabaergics } \\
\text { Antagonists }\end{array}$ & 0.6 \\
\hline
\end{tabular}

or inverse agonists. Availability of any of these classes of compounds as pharmacological tools can provide insight into the physiological role of the receptor.

MrgD, a member of the Mas-related gene family $[3,5]$, is specifically expressed in small diameter $\mathrm{IB}^{+}{ }^{+}$neurons in the dorsal root ganglion, an expression pattern that suggests that MrgD play a role in the initiation or modulation of pain signals and, as such, could be a novel target for the development of pain therapeutic agents. Elegant axonal tracer studies have shown that MrgD is expressed in a subpopulation of nonpeptidergic, nociceptive neurons that project exclusively to the skin. These neurons innervate a specific layer of the epidermis, distinct from that innervated by peptidergic nerve fibers [13]. To better understand the role of MrgD in pain, our group has undertaken studies examining MrgD modulation of ion channels thought to be involved in pain signal transduction. We have observed a novel interaction between MrgD and KCNQ/M-type potassium channels that results in suppression of these channels and enhancement of the excitability of DRG neurons [14]. These studies suggest that antagonism of MrgD could be beneficial for the treatment of pain. Genetic ablation of mouse neurons that express MrgD in adulthood caused specific deficits in the behavioral response to noxious mechanical stimuli but not to noxious heat or cold stimuli [15].

Beta-alanine has been identified as a ligand for MrgD [9]. Beta-alanine is a small amino acid structurally related to the major inhibitory neurotransmitters gamma-aminobutyric acid (GABA) and glycine. Our studies confirmed the previous finding that GABA, but not glycine, weakly stimulated cells expressing human and rat MrgD [9]. It has been reported that beta-alanine decreases glutamatergic excitation by binding to, and thus competing for, the glycine coagonist site of the $\mathrm{N}$-methyl-D-aspartate (NMDA) receptor $[16,17]$. It also has been shown that beta-alanine can activate glycine and GABA receptors, though with less efficacy than their native ligands $[18,19]$. As a result of the promiscuous interaction of beta-alanine with other receptors precise interpretation of the in vivo studies leading to insight regarding the role of beta-alanine in nociception is confounded. Another study was recently published in which a high-throughput calcium mobilization assay was used for the identification of agonists for MrgD and MrgA. Two agonists belonging to puridazine and thiazole families were identified as hits for MrgD [11]. Ligands for MrgA, another member of the Mrg family present only in rodents, were also reported in that study and are of interest from a selectivity perspective.
When reverse pharmacology approaches are employed in deorphaning, an open question is whether the ligand identified is natural or surrogate [20]. This concern also applies to beta-alanine. Local concentrations of beta-alanine previously have been reported to be $\sim 50 \mu \mathrm{M}$ in rat sciatic nerve and $60 \mu \mathrm{M}$ in cat brain $[21,22] . \mathrm{EC}_{50}$ values for $\mathrm{MrgD}$ expressing cell lines have been reported to range from $8.6 \mu \mathrm{M}$ [11], $15 \pm 1 \mu \mathrm{M}$ [9], and $57.8+3.5 \mu \mathrm{M}$ [20]. After extensive characterization of $\sim 100$ clones, we were successful in obtaining cell lines showing $\mathrm{EC}_{50}$ values in the low micromolar range. Having a ligand (irrespective of its being cognate or surrogate) and a sensitive cell line enabled us to develop a robust FLIPR assay with a $Z^{\prime}$ factor greater than 0.7 as an aid to identifying both agonists and antagonists from the same screen. In addition to maximizing the information that can be obtained about compounds screened, great cost savings are realized by combining the two screens into one. We validated the assay by screening the LOPAC collection. Although the time required to screen each plate, $\sim 10$ minutes, may be viewed as a shortcoming of this assay format, we view this as a relatively minor concern when balanced against the amount of data generated and cost savings from this assay.

In drug discovery, ideally, confirmation of hits identified from HTS is done using secondary assays with different end point read outs to eliminate false positive hits. To further characterize this cell line and explore its utility for other assays, we investigated $G$ protein coupling by measuring MrgD responses to beta-alanine after blocking PLC and Gi. Our results are in agreement with previous reports that in $\mathrm{CHO}$ cells, $\mathrm{MrgD}$ signaling occurs primarily through $\mathrm{Gq}[9,11,14]$. However, this observation may differ across cell types, since in DRG neurons, inhibition of the $\mathrm{M}$ current by beta-alanine activated MrgD occurs primarily through the Gi pathway [14]. Thus it is pivotal that interpretation of findings from a cell line is done with caution and $\mathrm{G}$ protein coupling is studied in native context.

To ensure that this dual-addition assay format can be applied to other GPCRs with a known agonist, we also tested this assay on MrgX1 (data not shown). These experiments confirmed that this assay format could be extended to additional GPCRs. In addition to its utility in screening new targets, this dual-addition assay format also can be extended to receptors such as mGluRs where both agonists and antagonists have been reported to have therapeutic value [23].

Antagonists identified from FLIPR often function as inverse agonists and can be characterized further by secondary assays. Having a repertoire of agonists, antagonists and inverse agonists would be extremely useful in efforts to validate MrgD. It was not surprising that most of the compounds obtained from the LOPAC screen had $\mathrm{EC}_{50}$ and $\mathrm{IC}_{50}$ values in the micromolar range. Compounds identified from this LOPAC screen have very limited utility primarily due to the lack of selectivity. Chlorpromazine and thioradizine are both first-generation typical antipsychotic drugs and produce catalepsy in rodents and extrapyramidal side effects in humans resulting from blockade of D2 receptors [24]. 
Chlorpromazine is often referred to as "dirty drug" because it acts on many receptors including dopaminergic (D1, D2, D3, and D4), serotonergic (5-HT1 and 5-HT2), histaminergic $\mathrm{H} 1$, alpha1/alpha2, and muscarinic (M1/M2) receptors [25]. Chlorpromazine is a weak inhibitor of dopamine reuptake resulting in mild antidepressive and antiparkinsonian effects. In addition, chlorpromazine inhibits calmodulindependent stimulation of cyclic nucleotide phosphodiesterase [26] and nitric oxide synthase [27]. Interestingly enough chlorpromazine has been used for analgesia in cancer patients as an adjunct to morphine in advanced cancer $[28,29]$.

Thioradizine is a phenothiazine antipsychotic used in the management of psychoses, including schizophrenia. However, increased risk of QTc prolongation [30] and sudden death in patients treated with thioridazine has been reported [31]. The cardiac effects of thioridazine are related to the concentration-dependent blockade of the cardiac-delayed inward rectifier potassium channel (Ikr) $[32,33]$. Thioridazine has recently attracted interest as a potential candidate for development as an antimicrobial drug. With the emergence of multidrug resistant bacteria, novel derivatives of thioridazine with reduced affinity for the Ikr channel and dopamine receptors but higher potency as antimicrobial have the potential to treat infectious diseases [34].

HA-966 is an excitatory amino acid antagonist, acting principally at the NMDA receptor subtype through a selective interaction with the glycine modulatory site of the receptor complex. Though the mechanism of action is not well understood, it has been suggested that NMDA antagonists/opioid combinations have promise as analgesic agents for the long-term treatment of pain [35].

Thus the antagonistic and agonist activities detected from screening LOPAC using our MrgD assay may be not be selective for $\mathrm{MrgD}$. It is also possible that some of these compounds from the LOPAC collection may be acting downstream of the receptor, inhibiting $\mathrm{Gi}, \mathrm{Gq}$ coupling or any other signaling intermediates. Further studies are needed to assess the specific mechanism of action of these compounds.

The primary objective of this study was to develop an assay for identification of agonists and antagonists from the same screen. The data presented here demonstrate that the dual-addition assay format can be successfully employed and adapted for HTS. Compounds identified here may have some utility as positive controls in future screening efforts. It is often the case in drug discovery that extensive medicinal chemistry efforts are required to obtain highly potent druglike compounds. Thus the compounds identified here may provide some insights for further chemistry or structurebased drug design efforts.

In conclusion, we have developed an HTS assay for $\mathrm{MrgD}$ that will enable the identification of both agonists and antagonists from a single screen and assist in the identification of pharmacological tools that will help elucidate the role of MrgD in pain. Our results demonstrated that the dual agonist/antagonist assay format is feasible and can be extended to most GPCRs with a known agonist.

\section{Competing Interests}

All authors were employees of Wyeth Research at the time the research was carried out.

\section{Acknowledgment}

The authors would like to acknowledge Robert Crozier and Brendan Bingham for a critical reading of the paper.

\section{References}

[1] J. P. Overington, B. Al-Lazikani, and A. L. Hopkins, "How many drug targets are there?" Nature Reviews Drug Discovery, vol. 5, no. 12, pp. 993-996, 2006.

[2] O. Civelli, Y. Saito, Z. Wang, H.-P. Nothacker, and R. K. Reinscheid, "Orphan GPCRs and their ligands," Pharmacology and Therapeutics, vol. 110, no. 3, pp. 525-532, 2006.

[3] X. Dong, S.-K. Han, M. J. Zylka, M. I. Simon, and D. J. Anderson, "A diverse family of GPCRs expressed in specific subsets of nociceptive sensory neurons," Cell, vol. 106, no. 5, pp. 619-632, 2001.

[4] P. M. C. Lembo, E. Grazzini, T. Groblewski et al., "Proenkephalin A gene products activate a new family of sensory neuron-specific GPCRs," Nature Neuroscience, vol. 5, no. 3, pp. 201-209, 2002.

[5] M. J. Zylka, X. Dong, A. L. Southwell, and D. J. Anderson, "Atypical expansion in mice of the sensory neuron-specific Mrg G protein-coupled receptor family," Proceedings of the National Academy of Sciences of the United States of America, vol. 100, no. 17, pp. 10043-10048, 2003.

[6] N. Robas, E. Mead, and M. Fidock, "MrgX2 is a high potency cortistatin receptor expressed in dorsal root ganglion," The Journal of Biological Chemistry, vol. 278, no. 45, pp. 4440044404, 2003.

[7] E. Grazzini, C. Puma, M.-O. Roy et al., "Sensory neuronspecific receptor activation elicits central and peripheral nociceptive effects in rats," Proceedings of the National Academy of Sciences of the United States of America, vol. 101, no. 18, pp. 7175-7180, 2004.

[8] S.-K. Han, X. Dong, J.-I. Hwang, M. J. Zylka, D. J. Anderson, and M. I. Simon, "Orphan G protein-coupled receptors Mrgal and Mrgcl1 are distinctively activated by RF-amide-related peptides through the G $\alpha$ q/11 pathway," Proceedings of the National Academy of Sciences of the United States of America, vol. 99, no. 23, pp. 14740-14745, 2002.

[9] T. Shinohara, M. Harada, K. Ogi et al., "Identification of a G protein-coupled receptor specifically responsive to $\beta$-alanine," The Journal of Biological Chemistry, vol. 279, no. 22, pp. 23559-23564, 2004.

[10] P. Kunapuli, S. Lee, W. Zheng et al., "Identification of small molecule antagonists of the human mas-related gene-X1 receptor," Analytical Biochemistry, vol. 351, no. 1, pp. 50-61, 2006.

[11] R. Zhang, P.-K. Yan, C.-H. Zhou, J.-Y. Liao, and M.-W. Wang, "Development of a homogeneous calcium mobilization assay for high throughput screening of mas-related gene receptor agonists," Acta Pharmacologica Sinica, vol. 28, no. 1, pp. 125131, 2007.

[12] S. P. Nawoschik, M. Olsen, D. L. Smith, and X. Khawaja, "Stable expression of adenylyl cyclase 2 leads to the functional rescue of human 5-HT6 receptor in a CHODUKX cell line," 
Journal of Pharmacological and Toxicological Methods, vol. 55, no. 3, pp. 323-331, 2007.

[13] M. J. Zylka, F. L. Rice, and D. J. Anderson, “Topographically distinct epidermal nociceptive circuits revealed by axonal tracers targeted to Mrgprd," Neuron, vol. 45, no. 1, pp. 17-25, 2005.

[14] R. A. Crozier, S. K. Ajit, E. J. Kaftan, and M. H. Pausch, "MrgD activation inhibits KCNQ/M-currents and contributes to enhanced neuronal excitability," Journal of Neuroscience, vol. 27, no. 16, pp. 4492-4496, 2007.

[15] D. J. Cavanaugh, H. Lee, L. Lo et al., "Distinct subsets of unmyelinated primary sensory fibers mediate behavioral responses to noxious thermal and mechanical stimuli," Proceedings of the National Academy of Sciences of the United States of America, vol. 106, no. 22, pp. 9075-9080, 2009.

[16] K. Ogita, T. Suzuki, and Y. Yoneda, "Strychnine-insensitive binding of $[3 \mathrm{H}]$ glycine to synaptic membranes in rat brain, treated with triton X-100," Neuropharmacology, vol. 28, no. 11, pp. 1263-1270, 1989.

[17] L. M. Pullan and R. J. Powel, "Comparison of binding at strychnine-sensitive (inhibitory glycine receptor) and strychnine-insensitive (N-methyl-D-aspartate receptor) glycine binding sites," Neuroscience Letters, vol. 148, no. 1-2, pp. 199-201, 1992.

[18] F. S. Wu, T. T. Gibbs, and D. H. Farb, "Dual activation of GABAA and glycine receptors by beta-alanine: inverse modulation by progesterone and 5 alpha-pregnan-3 alpha-ol20-one," The European Journal of Pharmacology, vol. 246, pp. 239-246, 1993.

[19] S. Rajendra, J. W. Lynch, and P. R. Schofield, "The glycine receptor," Pharmacology and Therapeutics, vol. 73, no. 2, pp. 121-146, 1997.

[20] Z. Wang, T. Takahashi, Y. Saito et al., "Salusin $\beta$ is a surrogate ligand of the mas-like G protein-coupled receptor MrgA1," European Journal of Pharmacology, vol. 539, no. 3, pp. 145150, 2006.

[21] N. Marks, R. K. Datta, and A. Lajtha, "Distribution of amino acids and of exo- and endopeptidases along vertebrate and invertebrate nerves," Journal of Neurochemistry, vol. 17, no. 1, pp. 53-63, 1970.

[22] H. H. Tallan, S. Moore, and W. H. Stein, "Studies on the free amino acids and related compounds in the tissues of the cat," The Journal of Biological Chemistry, vol. 211, no. 2, pp. 927939, 1954.

[23] Z.-Q. Yang, "Agonists and antagonists for group III metabotropic glutamate receptors 6, 7 and 8," Current Topics in Medicinal Chemistry, vol. 5, no. 9, pp. 913-918, 2005.

[24] S.-Y. Yang, Y.-H. Kao Yang, M.-Y. Chong, Y.-H. Yang, W.H. Chang, and C.-S. Lai, "Risk of extrapyramidal syndrome in schizophrenic patients treated with antipsychotics: a population-based study," Clinical Pharmacology and Therapeutics, vol. 81, no. 4, pp. 586-594, 2007.

[25] S. J. Peroutka and S. H. Snyder, "Relationship of neuroleptic drug effects at brain dopamine, serotonin, $\alpha$-adrenergic, and histamine receptors to clinical potency," American Journal of Psychiatry, vol. 137, no. 12, pp. 1518-1522, 1980.

[26] B. U. Raess and F. F. Vincenzi, "Calmodulin activation of red blood cell $\left(\mathrm{Ca}_{2}^{+}+\mathrm{Mg}^{+}\right)$-ATPase and its antagonism by phenothiazines," Molecular Pharmacology, vol. 18, no. 2, pp. 253-258, 1980.

[27] M. Palacios, J. Padron, L. Glaria et al., "Chlorpromazine inhibits both the constitutive nitric oxide synthase and the induction of nitric oxide synthase after LPS challenge,"
Biochemical and Biophysical Research Communications, vol. 196, no. 1, pp. 280-286, 1993.

[28] C. Drinkwater and R. G. Twycross, "Analgesia in terminal malignant disease," British Medical Journal, vol. 1, no. 6172, pp. 1201-1202, 1979.

[29] B. Mclver, D. Walsh, and K. Nelson, "The use of chlorpromazine for symptom control in dying cancer patients," Journal of Pain and Symptom Management, vol. 9, no. 5, pp. 341-345, 1994.

[30] E. P. Harrigan, J. J. Miceli, R. Anziano et al., "A randomized evaluation of the effects of six antipsychotic agents on QTc, in the absence and presence of metabolic inhibition," Journal of Clinical Psychopharmacology, vol. 24, no. 1, pp. 62-69, 2004.

[31] J. G. Reilly, S. A. Ayis, I. N. Ferrier, S. J. Jones, and S. H. L. Thomas, "Thioridazine and sudden unexplained death in psychiatric in-patients," British Journal of Psychiatry, vol. 180, pp. 515-522, 2002.

[32] B. Drolet, F. Vincent, J. Rail et al., "Thioridazine lengthens repolarization of cardiac ventricular myocytes by blocking the delayed rectifier potassium current," Journal of Pharmacology and Experimental Therapeutics, vol. 288, no. 3, pp. 1261-1268, 1999.

[33] H. Tie, B. D. Walker, C. B. Singleton et al., "Clozapine and sudden death," Journal of Clinical Psychopharmacology, vol. 21, no. 6, pp. 630-632, 2001.

[34] R. H. K. Thanacoody, A. K. Daly, J. G. Reilly, I. N. Ferrier, and S. H. L. Thomas, "Factors affecting drug concentrations and QT interval during thioridazine therapy," Clinical Pharmacology and Therapeutics, vol. 82, no. 5, pp. 555-565, 2007.

[35] A. C. Foster and J. A. Kemp, "HA-966 antagonizes N-methyl$\mathrm{D}$-aspartate receptors through a selective interaction with the glycine modulatory site," Journal of Neuroscience, vol. 9, no. 6, pp. 2191-2196, 1989. 

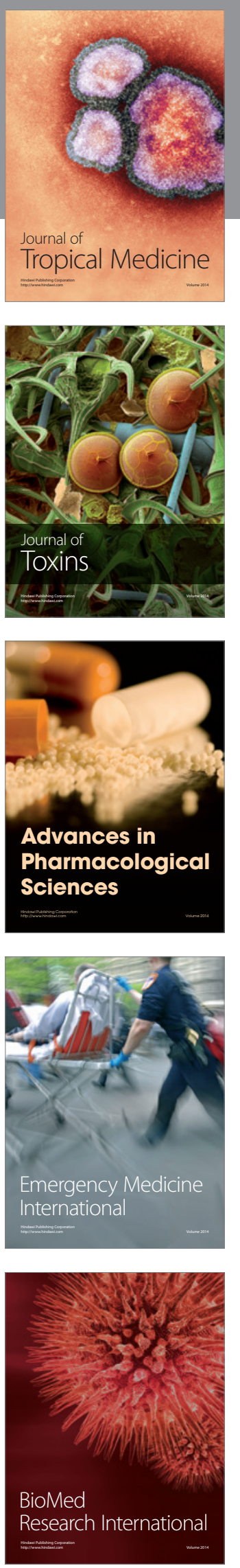
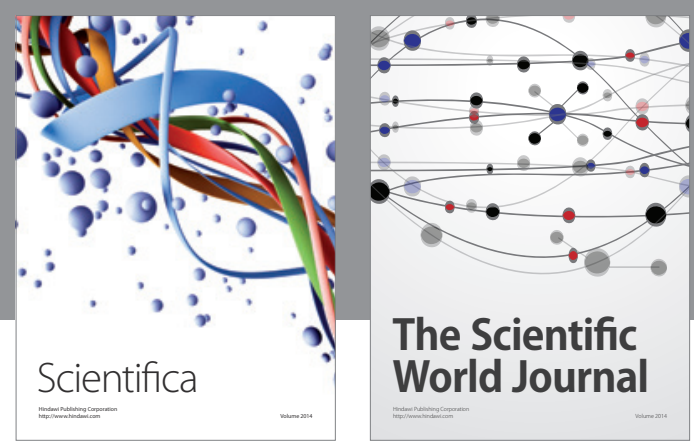

The Scientific World Journal
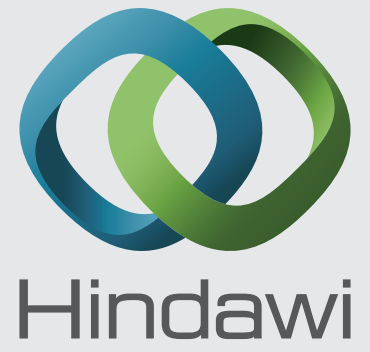

Submit your manuscripts at

http://www.hindawi.com
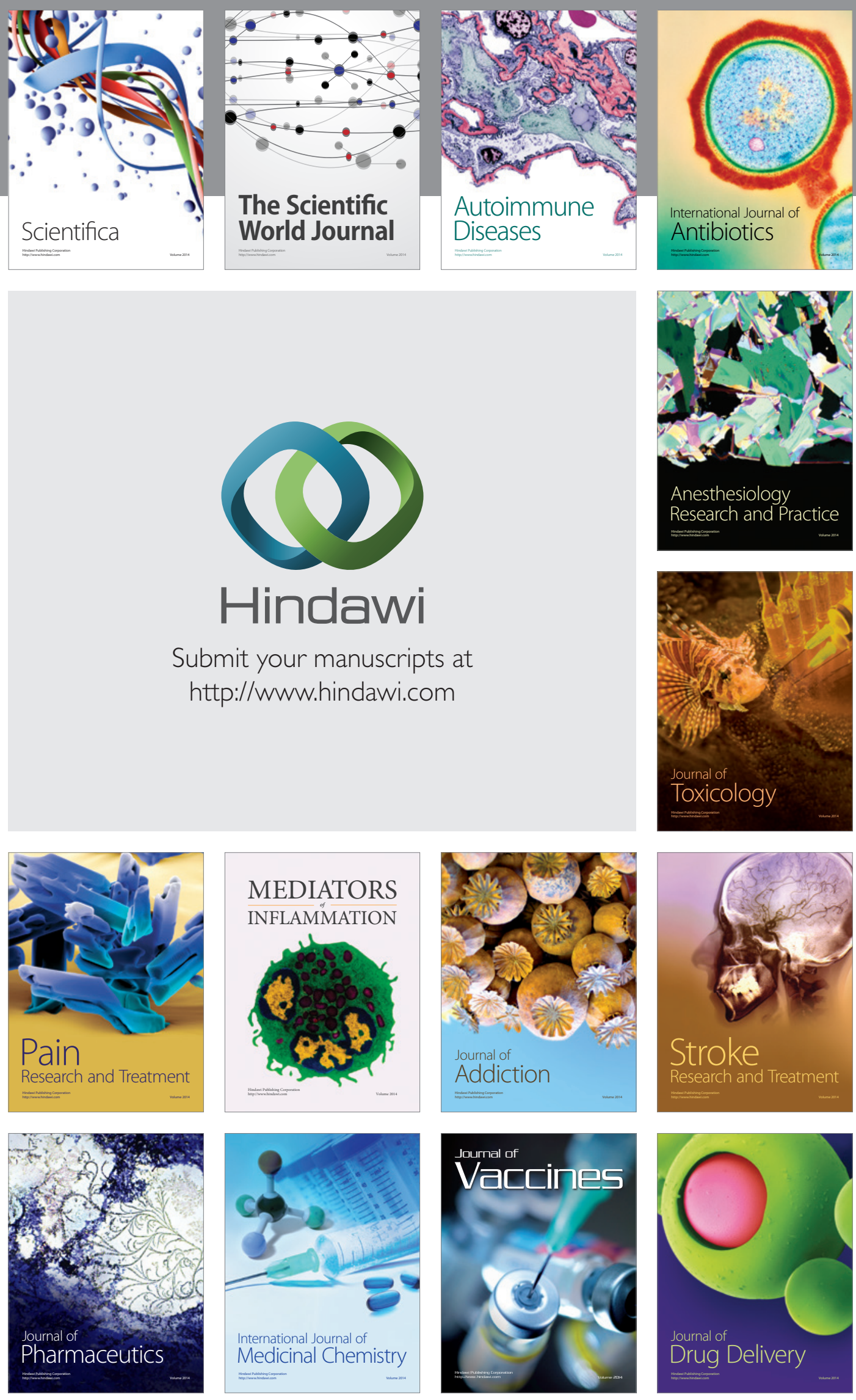